\title{
"Karlsruher Forum Ethik in Recht und Technik" gegründet
}

ins Internet. Bereits erschienen sind Ausgaben zu den Themen: 5 Jahre nach Rio, ökologische Unternehmenskommunikation und ökologische Produktpolitik; das aktuelle Magazin widmet sich dem Thema neue Mobilitätsdienstleistungen. Auch der Vermittlung von Zukunftswissen widmet sich Z_punkt: Derzeit bereitet das Institut einen Bild-Text-Band zu vergangenen und aktuellen Visionen der Zukunft vor, das Thema wird auch Gegenstand einer Galerie im Internet von Ekkehard Fulda, ITAS

Anfang dieses Jahres wurde das "Karlsruhe Forum Ethik in Recht und Technik" gegründet. Das Forum, dem Vertreter aus Wissenschaft, Wirtschaft und Politik angehören, will Gewicht im gesellschaftlichen Diskurs über Technik gewinnen.

Karlsruhe verfügt über höchste rechtliche ("Residenz des Rechts") und herausragende technische wie auch natur- und technikwissenschaftliche Kompetenz ("Technologieregion" mit hervorragenden Hochschulen und Forschungsinstitutionen). Auf diesen Gedanken aufbauend wurde Anfang April das "Karlsruher Forum Ethik in Recht und Technik" als eingetragener Verein gegründet. Ziel dieses Forums ist es, den gesellschaftlichen Dialog über Technik zu fördern, und zwar in einer breiten und allgemein 
verständlichen Weise. Zur Sprache kommen sollen dabei insbesondere die ethischen und rechtlichen Aspekte gegenwärtiger und erwartbarer Technologien, wobei wirtschaftliche, soziale, kommunikative und etwa ökologische Gesichtspunkte einzubeziehen sind. Die gesellschaftliche Akzeptanz moderner Technik kann so gestärkt - oder aber ihre Grenzen aufgezeigt werden.

Als Auftaktveranstaltung ist für das Frühjahr 1999 eine Tagung mit namhaftenVertretern von Wissenschaft, Wirtschaft und Politik vorgesehen über die Thematik "Technikangst Wege aus der deutschen Befindlichkeit". Die Initiatoren streben eine mindestens bundesweite Ausstrahlung der Veranstaltung an; das Karlsruher Forum will Gewicht im gesellschaftlichen Diskurs gewinnen, indem es als hochkarätiges Wissenschaftsforum Probleme aufgreift, die im Spannungsfeld zwischen Ethik, Recht und Technik stehen. Dabei wird dem Forum ein Kuratorium zur Seite stehen, dem Persönlichkeiten von nationalem Rang aus den Bereichen Recht, Technik oder Ethik angehören. So haben die Präsidentin des Bundesverfassungsgerichts, Professor Dr. Jutta Limbach, der Generalbundesanwalt, Kay Nehm, der Präsident des Bundesgerichtshofs, Karlmann Geiß sowie der Bundesminister des Auswärtigen, Dr. Klaus Kinkel, ihre Mitgliedschaft in dem Kuratorium des Karlsuher Forums bereits zugesagt.

Gründungsmitglieder des "Karlsruher Forum Ethik in Recht und Technik e.V." sind: die Stadt Karlsruhe selbst, das Forschungszentrum Karlsruhe, die Landeskreditbank Baden-Württemberg, die Universität Karlsruhe, die Industrie- und Handelskammer Karlsruhe, das Badenwerk sowie das Zentrum für Kunst und Medientechnologie. Der Karlsruher Oberbürgermeister, Professor Dr. Gerhard Seiler, ist Vorsitzender des Trägervereins, den stellvertretenden Vorsitz hat Professor Dr. Manfred Popp inne, Vorstandsvorsitzender des Forschungszentrums Karlsruhe, drittes Vorstandsmitglied und Schatzmeister des Vereins ist Michael Gaßner, Prokurist des Badenwerks. Die Geschäftsstelle des Forums wird im Stadtbüro des Forschungszentrums Karlsruhe in der Weberstraße 5 eingerichtet.

\section{Kontakt}

Dr. Ekkehard Fulda

Karlsruher Forum Ethik in Recht und Technik

Weberstr. 5, D-76133 Karlsruhe

Tel.: + 49 (0) 7247/82-4644

Fax: + 49 (0) 721/843167

E-mail: Ekkehard.Fulda@t-online.de 